\title{
Chapter 6 \\ Biomarkers for Immune Modulatory Treatment in Head and Neck Squamous Cell Carcinoma (HNSCC)
}

\author{
Danny Rischin
}

\section{Background}

Immune checkpoint inhibitors have changed the standard of care in recurrent/metastatic mucosal head and neck squamous cell carcinoma. Initially in the 2 nd-line post platinum based chemotherapy setting $[1,2]$, and more recently based on the results of the Keynote-048 trial in the 1st-line R/M setting [3]. Although responses can be durable, only a minority of patients respond. Hence, the need for predictive markers to ensure these therapies are provided to patients most likely to benefit, whilst sparing patients who are unlikely to benefit from these treatments.

\section{Potential Predictive Biomarkers for Immune Checkpoint Inhibitors}

1. Immune checkpoint ligand expression e.g., programmed death-ligand 1 (PD-L1)

2. Markers of a T-cell inflamed microenvironment e.g., gene expression profiles

3. Markers of tumour neoepitope burden e.g., tumour mutation burden

4. Multidimensional quantitative immunohistochemistry (IHC)/immunofluorescence (IF) e.g., PD1/PD-L1

D. Rischin $(\bowtie)$

Department of Medical Oncology and Sir Peter MacCallum Department of Oncology,

Peter MacCallum Cancer Centre and University of Melbourne, Melbourne, VIC, Australia

e-mail: danny.rischin@ petermac.org 


\section{PD-L1}

PD-L1 is the most studied predictive biomarker for response to immune checkpoint inhibitors. However, the field has been hampered by a number of factors including the use of different antibodies, measurement of tumour versus immune cells versus both, variable scoring criteria, and variable expression and cut-offs across tumour types [4]. In Table 6.1, the antibodies employed for some of the more common immune checkpoint inhibitors in use are shown, as well as the cells scored. With regard to staining tumour cells the antibodies behave similarly, with the exception of SP142 that stains a lower percentage of cells. However, in general these antibodies are not interchangeable, and it is best to use the same antibody and ideally the same assay as was used in the relevant trial in that cancer [4]. In general, good reproducibility has been demonstrated for scoring of tumour cells, but this is not the case for measuring immune cells only [5].

In R/M HNSCC there does seem to be a correlation between PD-L1 expression and response, as well as survival, albeit not in all studies (Table 6.2). In R/M HNSCC, as in other cancers, a range of assays and scoring criteria has been used. However, in this manuscript the focus will largely be on the assays used in the key pembrolizumab

Table 6.1 PD-L1 antibodies

\begin{tabular}{l|l|l|l|l}
\hline & Nivolumab & Pembrolizumab & Durvalumab & Atezolizumab \\
\hline $\begin{array}{l}\text { Primary } \\
\text { antibody }\end{array}$ & $28-8$ & $22 \mathrm{C} 3$ & SP263 & SP142 \\
\hline Scoring & Tumour & $\begin{array}{l}\text { Tumour - TPS } \\
\text { Tumour } \\
+ \text { immune cells - CPS }\end{array}$ & Tumour & $\begin{array}{l}\text { Tumour and immune } \\
\text { cells }\end{array}$ \\
\hline
\end{tabular}

Table 6.2 Correlation between PD-L1 expression and response in R/M HNSCC

\begin{tabular}{l|l|l|l|l}
\hline $28-8$ & $\begin{array}{l}\text { PD-L1 } \geq 1 \% \\
\text { TC }\end{array}$ & PD-L1 $<1 \%$ & & \\
\hline Nivolumab & $\mathbf{1 8 \%}(\mathrm{n}=96)$ & $\mathbf{1 2 \%}(\mathrm{n}=76)$ & & Ferris et al. [6] \\
\hline SP263 & $\begin{array}{l}\text { PD-L1 } \geq 25 \% \\
\text { TC }\end{array}$ & $\begin{array}{l}\text { PD-L1 }<25 \% \\
\text { TC }\end{array}$ & & \\
\hline Durvalumab & $\mathbf{1 6 \%}(\mathrm{n}=112)$ & $\mathbf{9 \%}(\mathrm{n}=67)$ & & $\begin{array}{l}\text { Zandberg et al. [7], Siu } \\
\text { et al. [8] }\end{array}$ \\
\hline SP142 & $\begin{array}{l}\text { PD-L1 } \\
\text { IC } \geq 5 \%\end{array}$ & PD-L1 IC $<5 \%$ & & \\
\hline Atezolizumab & $\mathbf{2 4 \%}(\mathrm{n}=25)$ & $\mathbf{1 4 \%}(\mathrm{n}=7)$ & & Colevas et al. [9] \\
\hline 22 C3 & $\begin{array}{l}\text { PD-L1 } \\
\text { CPS } \geq 20\end{array}$ & PD-L1 CPS $\geq 1$ & PD-L1 & \\
\hline Pembrolizumab & & $\mathbf{2 2 \%}(\mathrm{n}=127)$ & $\mathbf{4 \%}(\mathrm{n}=25)$ & Chow et al. [10] \\
\hline Pembrolizumab & $\mathbf{2 3 \%}(\mathrm{n}=133)$ & $\mathbf{1 9 \%}(\mathrm{n}=257)$ & $\mathbf{4 \%}(\mathrm{n}=44)$ & Burtness et al. [3] \\
\hline Pembrolizumab & TPS $\geq 50 \%$ & TPS $<50 \%$ & & \\
\hline
\end{tabular}


trials in R/M HNSCC that have led to approvals based on PD-L1 expression. In the 1st-line setting worldwide and in the 2nd-line setting in Europe use of pembrolizumab for R/M HNSCC first requires evaluation of PD-L1 expression.

The first phase 3 trial in R/M HNSCC was the trial of nivolumab versus standard of care (investigators choice-methotrexate, docetaxel or cetuximab) [1]. Patients were enrolled regardless of PD-L1 expression, and it was also not a stratification factor. Based on this trial nivolumab was approved for treatment in platinum resistant R/M HNSCC in all-comers, i.e., no restriction based on PD-L1 expression. In an exploratory analysis, tumour PD-L1 expression did not appear to be predictive of benefit [6].

In the pembrolizumab trials in HNSCC the PD-L1 22C3 pharmDx companion diagnostic assay has been used. Two scoring methods are available:

1. The tumour proportion score (TPS), which is the percentage of viable tumour cells with partial or complete membrane staining at any intensity

2. The combined positive score (CPS), which is the ratio of the number of PD-L1expressing cells (tumour cells, lymphocytes, macrophages) to the number of all viable tumour cells $\times 100$

The PD-L1 CPS score has been shown to have good reproducibility in a gastric cancer study [11]. In an exploratory analysis of the Keynote-012 HNSCC cohort, measurement of tumour + immune cells seemed to be more predictive of response to pembrolizumab than measurement of tumour cells only [10]. In the Keynote-040 trial pembrolizumab was compared to standard of care (investigators choicemethotrexate, docetaxel or cetuximab) in patients who had received prior platinum [2]. Eligibility required submission of a tissue sample for PD-L1 assessment and PD-L1 TPS ( $\geq 50 \%$ vs. $<50 \%$ ) was a stratification factor. $26 \%$ of the population had PD-L1 TPS $\geq 50 \%$, and when analysed by TPS scores the benefit of pembrolizumab appeared to be predominantly in this population. In Europe, the EMA approved pembrolizumab for platinum pre-treated HNSCC in patients with PD-L1 TPS $\geq 50 \%$. In an exploratory analysis when analysed by PD-L1 CPS ( $\geq 1 \%$ vs. $<1 \%$ ) $83 \%$ of the population had CPS $\geq 1$, and it was predictive of benefit.

There has been a preliminary report of a posthoc analysis of efficacy outcomes based on PD-L1 scoring techniques in Keynote-040 [12]. Standard receiver operating characteristic curves were generated for TPS and CPS for patients receiving pembrolizumab versus SOC to demonstrate the relationship between pembrolizumab and SOC at each cutoff. Concordance between TPS and CPS cutoffs was $77 \%$ at a cutoff of $1,91 \%$ at a cutoff of 20 , and $95 \%$ at a cutoff of 50 . At lower expression levels, CPS detects a larger fraction of pembrolizumab responders than TPS while maintaining similar survival results. At higher expression levels, CPS $\geq 50$ can be used interchangeably with TPS $\geq 50 \%$. Based on these results it was concluded that CPS is a valid scoring method for determining PD-L1 status in patients with HNSCC.

The Keynote-048 trial evaluated the role of pembrolizumab alone or in combination with platinum-5-FU chemotherapy compared to the standard of care, the Extreme regimen of platinum, 5FU and cetuximab in patients receiving 1st-line 
systemic therapy for R/M HNSCC [3]. PD-L1 expression based on TPS ( $\geq 50 \%$ vs. $<50 \%$ ) was a stratification factor, with $22 \%$ having TPS $\geq 50 \%$. Key populations for the primary, secondary and exploratory endpoints were defined by PD-L1 CPS scores: $\geq 20, \geq 1$ and the total population. $40-45 \%$ of the population had CPS $\geq 20$ and $85 \%$ had CPS $\geq 1$. In this trial pembrolizumab monotherapy was shown to be superior to the Extreme regimen in the CPS $\geq 20$ and $\geq 1$ populations but not in the total population. The combination of pembrolizumab with chemotherapy was shown to be superior to the Extreme regimen in all three populations. For both monotherapy and the combination with chemotherapy there was increasing benefit (overall survival and response) with increasing PD-L1 CPS. Based on the Keynote-048 results, pembrolizumab approvals for use in the 1st-line R/M setting have been contingent on tumour PD-L1 expression as assessed by the CPS. The FDA restricted approval of pembrolizumab monotherapy to patients whose tumours express PD-L1 $\geq 1$, but approved the combination with platinum and 5FU for the total population. The EMA approved both monotherapy and the combination with platinum and 5FU for 1st-line treatment of metastatic or unresectable R/M HNSCC in adults whose tumours express PD-L1 with a CPS $\geq 1$.

PD-L1 expression using CPS enriches for the population likely to benefit from use of an immune checkpoint inhibitor, and can identify a population unlikely to derive much benefit. However, it is a weak predictor of benefit as only a minority of patients with PD-L1 CPS $\geq 20$ achieve a response and prolonged survival.

\section{Gene Expression Profiling}

Several signatures have been identified that are predictive of response to immune checkpoint inhibitors. These include the 'Teff' signature [13] defined by three genes (PD-L1, CXCL9, and IFN $\gamma$ ) and is associated with responses to atezolizumab in patients with non-small cell lung cancer and the 'T-cell inflamed gene expression profile' (GEP) [14] consisting of 18 interferon-gamma responsive genes and is associated with responses to pembrolizumab in melanoma and 9 different solid tumors, including HNSCC.

The T-cell inflamed GEP score is higher in patients with HNSCC who responded to pembrolizumab, with area under the receiver operating characteristic curve of 0.768 [15]. GEP was significantly correlated with PD-L1 expression in HNSCC $(\mathrm{r}=0.51)$, which is consistent with PD-L1 expression regulation by T-cell derived IFN $\gamma$. 


\section{Tumour Mutation Burden}

There is a correlation between the median mutation burden of a given tumour type and the probability of response to an immune checkpoint inhibitor [16]. HNSCC has a moderate TMB, with median number of coding somatic mutations per megabase of 5.0 [17]. TMB of HNSCC is similar to oesophago-gastric and urothelial tumours, and considerably lower than more responsive tumours such as melanoma, cutaneous SCC and mismatch repair deficient tumours.

In HNSCC patients treated with pembrolizumab, TMB is higher in responders, with area under the receiver operating characteristic curve of 0.617 [15]. There was no correlation between TMB and either GEP or PD-L1. In another study higher TMB was associated with benefit from anti-PD1/PD-L1 in HPV negative HNSCC [18].

\section{Combination of Tumour Mutation Burden and Gene Expression Profiling}

The combination of TMB and GEP had joint predictive utility in identifying HNSCC responders and non-responders to pembrolizumab in a study of 105 patients [15]. There were no responders in the patients with low TMB and low GEP, and only one responder in the group with high TMB but low GEP. The highest response rate was in the group with both high TMB and high GEP - 37\%. The group with low TMB but high GEP had an intermediate response rate of $16 \%$.

\section{Multidimensional Quantitative IHC/IF}

A recent meta-analysis compared biomarker modalities for predicting response to immune checkpoint blockade [19]. It concluded that modalities that permit assessment of more than one biomarker were promising, for instance multiplex immunohistochemistry or immunofluorescence e.g., evaluating PD1 to PD-L1 proximity. These strategies may improve the positive predictive value that remains low with single modality predictive biomarkers. These techniques take into account the spatial importance of tumour immune interactions and the contribution of protein marker co-expression. The area under the receiver operating characteristic curve for multiplex IHC/IF was 0.79 that was considerably higher than for single factors e.g., PD-L1, GEP or TMB. 


\section{Tissue Resident Memory $\left(\mathbf{T}_{\mathrm{RM}}\right)$ Cells}

Tissue resident memory cells are a subset of $\mathrm{T}$ cells that occupy tissues without recirculating. They are characterised by expression of CD103 and CD69 and are usually $\mathrm{CD} 8$ and $\mathrm{CD} 4$ positive. $\mathrm{T}_{\mathrm{RM}} \mathrm{s}$ have a role in infections and cancer immunosurveillance [20].

High levels of intratumoural CD103 positive immune cells $(\geq 30 \%)$ in patients with HPV associated oropharyngeal cancer treated predominantly with chemoradiation is associated with an excellent outcome independent of stage [21]. This was demonstrated in a retrospective training cohort from the Peter MacCallum Cancer Centre with a hazard ratio of $0.13(95 \%$ CI $0.02-0.94, \mathrm{P}=0.004)$ and confirmed in an independent validation cohort from the Princess Alexandra Hospital with a hazard ratio of $0.16(95 \% \mathrm{CI} 0.02-1.22, \mathrm{P}=0.02)$. The 5 year survival estimates for the patients with high intratumoural CD103 was $100 \%$ in both cohorts while in the patients with low $(<30 \%$ ) intratumoural CD103 it was $82 \%$ and $88 \%$ (Fig. 6.1pooled results from the two cohorts). In both cohorts, which were unselected i.e., contained both low and high risk HPV OPSCC, the CD103 high group represented $20 \%$ of the population.

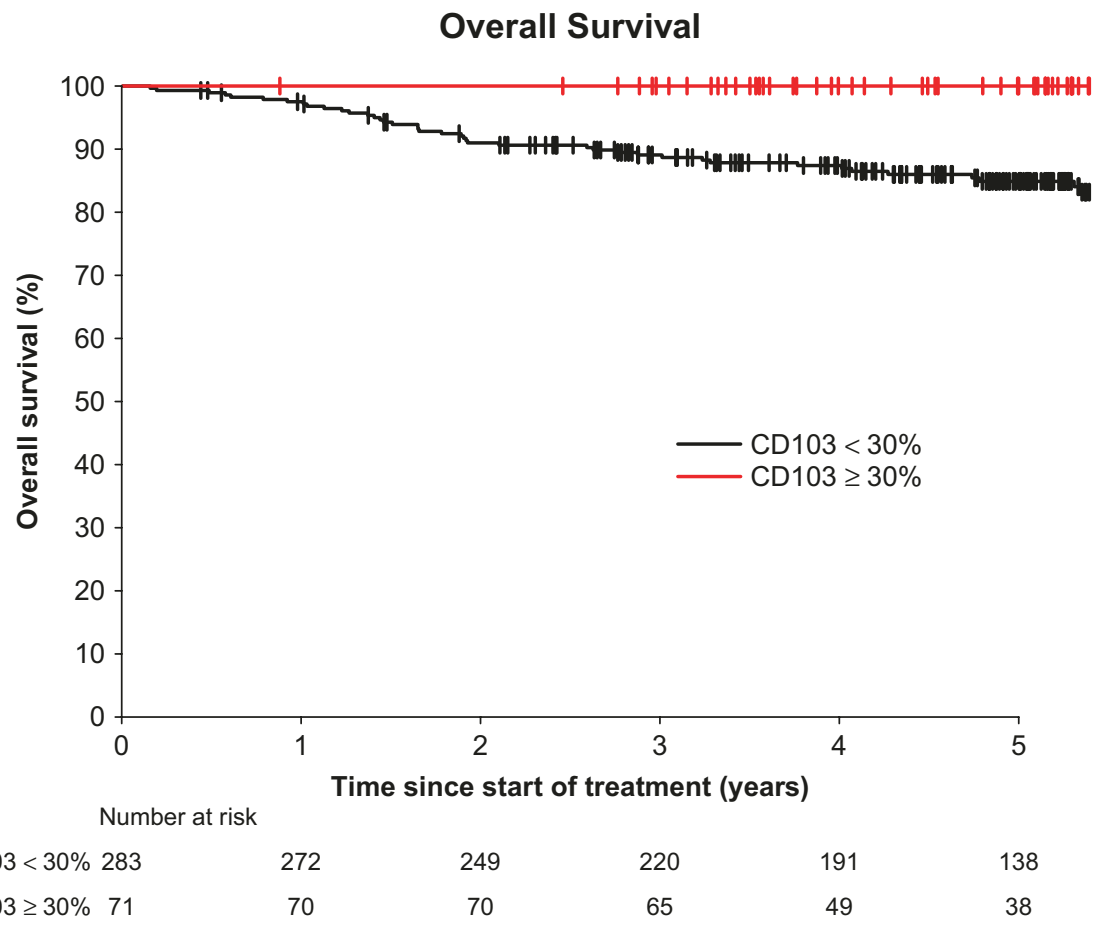

Fig. 6.1 Overall survival by intratumoural CD103 expression in locoregionally advanced HPV oropharyngeal SCC (combined Peter MacCallum and Princess Alexandra cohorts) 
Tumours with CD103+ cells co-expressed CD69 and CD8 on multispectral immunofluorescence consistent with $\mathrm{T}_{\mathrm{RM}} \mathrm{S}$. Tumours with high intratumoural CD103 also had higher expression of genes identified in a single cell gene expression analysis of $\mathrm{T}_{\mathrm{RM}} \mathrm{S}$ [22], as well as gene signatures associated with responses to pembrolizumab [14] and atezolizomab [13].

In another study there was expansion of CD103+ cells in biopsies of melanoma patients early during treatment with an anti-PD1 agent, which was greater in responding patients [23]. It has been suggested that high levels of CD103 CD8 tumour infiltrating lymphocytes in non-small cell lung cancer may be a predictive biomarker for sensitivity to immune checkpoint blockade [24]. It is reasonable to speculate that patients with CD103 + HPV-associated oropharyngeal cancer may be particularly sensitive to immune checkpoint blockade. While this population would be expected to be rare in the recurrent/metastatic setting, this locoregionally advanced population would be ideal candidates for de-escalation strategies in general, and in particular de-escalation trials that incorporate immune checkpoint blockade.

\section{Conclusion}

For the first time in HNSCC we have treatments approved based on the results of a companion diagnostic. Pembrolizumab for first-line treatment of recurrent metastatic disease requires assessment of PD-L1 expression as measured by the CPS. The PD-L1 CPS score enriches for populations more likely to respond, but the false positive predictive value remains high. Better predictive biomarkers are required, and while some show promise, clinical utility in HNSCC has not been established.

\section{References}

1. Ferris RL, Blumenschein G Jr, Fayette J, Guigay J, Colevas AD, Licitra L, et al. Nivolumab for recurrent squamous-cell carcinoma of the head and neck. N Engl J Med. 2016;375(19):1856-67.

2. Cohen EEW, Soulieres D, Le Tourneau C, Dinis J, Licitra L, Ahn MJ, et al. Pembrolizumab versus methotrexate, docetaxel, or cetuximab for recurrent or metastatic head-and-neck squamous cell carcinoma (KEYNOTE-040): a randomised, open-label, phase 3 study. Lancet. 2019;393(10167):156-67.

3. Burtness B, Harrington KJ, Greil R, Soulieres D, Tahara M, de Castro G Jr, et al. Pembrolizumab alone or with chemotherapy versus cetuximab with chemotherapy for recurrent or metastatic squamous cell carcinoma of the head and neck (KEYNOTE-048): a randomised, open-label, phase 3 study. Lancet. 2019;394(10212):1915-28.

4. Torlakovic E, Lim HJ, Adam J, Barnes P, Bigras G, Chan AWH, et al. "Interchangeability" of PD-L1 immunohistochemistry assays: a meta-analysis of diagnostic accuracy. Mod Pathol. 2020;33(1):4-17. 
5. Reisenbichler ES, Han G, Bellizzi A, Bossuyt V, Brock J, Cole K, et al. Prospective multiinstitutional evaluation of pathologist assessment of PD-L1 assays for patient selection in triple negative breast cancer. Mod Pathol. 2020.

6. Ferris RL, Blumenschein G Jr, Fayette J, Guigay J, Colevas AD, Licitra L, et al. Nivolumab vs investigator's choice in recurrent or metastatic squamous cell carcinoma of the head and neck: 2-year long-term survival update of CheckMate 141 with analyses by tumor PD-L1 expression. Oral Oncol. 2018;81:45-51.

7. Zandberg DP, Algazi AP, Jimeno A, Good JS, Fayette J, Bouganim N, et al. Durvalumab for recurrent or metastatic head and neck squamous cell carcinoma: results from a single-arm, phase II study in patients with $\geq 25 \%$ tumour cell PD-L1 expression who have progressed on platinum-based chemotherapy. Eur J Cancer. 2019;107:142-52.

8. Siu LL, Even C, Mesia R, Remenar E, Daste A, Delord JP, et al. Safety and efficacy of Durvalumab with or without tremelimumab in patients with PD-L1-low/negative recurrent or metastatic HNSCC: the phase 2 CONDOR randomized clinical trial. JAMA Oncol. 2019;5(2):195-203.

9. Colevas AD, Bahleda R, Braiteh F, Balmanoukian A, Brana I, Chau NG, et al. Safety and clinical activity of atezolizumab in head and neck cancer: results from a phase I trial. Ann Oncol. 2018;29(11):2247-53.

10. Chow LQM, Haddad R, Gupta S, Mahipal A, Mehra R, Tahara M, et al. Antitumor activity of pembrolizumab in biomarker-unselected patients with recurrent and/or metastatic head and neck squamous cell carcinoma: results from the phase Ib KEYNOTE-012 expansion cohort. J Clin Oncol. 2016;34(32):3838-45.

11. Kulangara K, Zhang N, Corigliano E, Guerrero L, Waldroup S, Jaiswal D, et al. Clinical utility of the combined positive score for programmed death ligand-1 expression and the approval of pembrolizumab for treatment of gastric cancer. Arch Pathol Lab Med. 2019;143(3):330-7.

12. Cohen E, Harrington K, Soulières D, Le Tournea C, Licitra LF, Burtness B, Bal T, Juco J, Aurora-Garg D, Huang L, Swaby RF, Emancipator K. Analysis of efficacy outcomes based on programmed death ligand 1 (PD-L1) scoring techniques in patients with head and neck squamous cell carcinoma (HNSCC) from KEYNOTE-040. Ann Oncol. 2019;30(Suppl 5):v449-v74.

13. Kowanetz MZW, Mccleland M, et al. Pre-existing immunity measured by Teff gene expression in tumor tissue is associated with Atezolizumab efficacy in NSCLC. J Thorac Oncol. 2017;12:S1817-S8.

14. Ayers M, Lunceford J, Nebozhyn M, Murphy E, Loboda A, Kaufman DR, et al. IFNgamma-related mRNA profile predicts clinical response to PD-1 blockade. J Clin Invest. 2017;127(8):2930-40.

15. Cristescu R, Mogg R, Ayers M, Albright A, Murphy E, Yearley J, et al. Pan-tumor genomic biomarkers for PD-1 checkpoint blockade-based immunotherapy. Science. 2018;362(6411).

16. Yarchoan M, Hopkins A, Jaffee EM. Tumor mutational burden and response rate to PD-1 inhibition. N Engl J Med. 2017;377(25):2500-1.

17. Chalmers ZR, Connelly CF, Fabrizio D, Gay L, Ali SM, Ennis R, et al. Analysis of 100,000 human cancer genomes reveals the landscape of tumor mutational burden. Genome Med. 2017;9(1):34.

18. Hanna GJ, Lizotte P, Cavanaugh M, Kuo FC, Shivdasani P, Frieden A, et al. Frameshift events predict anti-PD-1/L1 response in head and neck cancer. JCI Insight. 2018;3(4).

19. Lu S, Stein JE, Rimm DL, Wang DW, Bell JM, Johnson DB, et al. Comparison of biomarker modalities for predicting response to PD-1/PD-L1 checkpoint blockade: a systematic review and meta-analysis. JAMA Oncol. 2019;5:1195.

20. Mueller SN, Mackay LK. Tissue-resident memory T cells: local specialists in immune defence. Nat Rev Immunol. 2016;16(2):79-89.

21. Solomon B, Young RJ, Bressel M, Cernelc J, Savas P, Liu H, et al. Identification of an excellent prognosis subset of human papillomavirus-associated oropharyngeal cancer patients by quantification of intratumoral CD103+ immune cell abundance. Ann Oncol. 2019;30(10):1638-46. 
22. Savas P, Virassamy B, Ye C, Salim A, Mintoff CP, Caramia F, et al. Single-cell profiling of breast cancer $\mathrm{T}$ cells reveals a tissue-resident memory subset associated with improved prognosis. Nat Med. 2018;24(7):986-93.

23. Edwards J, Wilmott JS, Madore J, Gide TN, Quek C, Tasker A, et al. CD103(+) tumorresident $\mathrm{CD} 8(+) \mathrm{T}$ cells are associated with improved survival in immunotherapy-naive melanoma patients and expand significantly during anti-PD-1 treatment. Clin Cancer Res. 2018;24(13):3036-45.

24. Wang P, Huang B, Gao Y, Yang J, Liang Z, Zhang N, et al. CD103(+)CD8(+) T lymphocytes in non-small cell lung cancer are phenotypically and functionally primed to respond to PD-1 blockade. Cell Immunol. 2018;325:48-55.

Open Access This chapter is licensed under the terms of the Creative Commons Attribution 4.0 International License (http://creativecommons.org/licenses/by/4.0/), which permits use, sharing, adaptation, distribution and reproduction in any medium or format, as long as you give appropriate credit to the original author(s) and the source, provide a link to the Creative Commons license and indicate if changes were made.

The images or other third party material in this chapter are included in the chapter's Creative Commons license, unless indicated otherwise in a credit line to the material. If material is not included in the chapter's Creative Commons license and your intended use is not permitted by statutory regulation or exceeds the permitted use, you will need to obtain permission directly from the copyright holder.

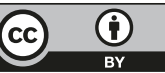

